\title{
Lo urbano desde la antropología en San Marcos
}

Recibido: 14/10/2016

Aprobado: 21/11/2016

\author{
Nekson Pimentel Sánchez \\ Universidad Nacional Mayor de San Marcos \\ < neksonunmsm@hotmail.com >
}

\begin{abstract}
RESUMEN
El presente documento pretende realizar un estado del arte sobre los estudios de lo urbano desde la antropología en la Universidad Nacional Mayor de San Marcos (San Marcos, en adelante), mediante el análisis de investigaciones empíricas que comprenden las tesis de Bachiller, Licenciatura, Maestría y Doctorado, desde las primeras décadas hasta 2012, que en conjunto forman parte del proceso de constitución y consolidación institucional de la antropología en la Decana de América. Incluye las diferentes temáticas desarrolladas que obedecen a nuestra propia inserción profesional y académica, las influencias teóricas y metodológicas en cada una de las producciones, así como las características de las investigaciones en las diversas etapas.
\end{abstract}

Palabras Clave: antropología en San Marcos, urbano, ciudad, campo y migración.

\section{The urban from the anthropology in San Marcos}

\begin{abstract}
This article intends to make a state of the art about the studies of the urban from the anthropology at the National University of San Marcos (San Marcos, hereinafter), through the analysis of empirical investigations that include the theses of Bachiller, Licenciatura, Mastership and Doctorate from the first decades to 2012, which together form part of the process of constituting and consolidating anthropology in the Dean of America. It includes the different topics developed that obey our own professional and academic insertion, the theoretical and methodological influences in each of the productions, as well as the characteristics of the research in the different stages.
\end{abstract}

KeYwords: Anthropology in San Marcos, urban, city, countryside and migration. 


\section{Estados del arte precedentes}

E xisten importantes balances desde las ciencias sociales sobre los estudios urbanos en el Perú, publicados en revistas, compilaciones y libros. Sin embargo, solo algunos dan cuenta sobre el lugar de la antropología en este campo, y ninguno propiamente acerca del abordaje de lo urbano por la antropología en San Marcos. A esto se suma la reducida cantidad de balances realizados desde la antropología.

Uno de los primeros balances fue realizado por Teófilo Altamirano, publicado hace más de tres décadas en la compilación de Humberto Rodríguez (1985), donde identifica y describe las tendencias en antropológica urbana y las temáticas desarrolladas por cada una de ellas hasta la década del ochenta. Posteriormente se han escrito otros balances, sobre todo desde la sociología. Así, encontramos los trabajos de Abelardo Sánchez (1990), Roger Iziga (1993), Max Meneses (1998), incorporado en su tesis de doctorado (1989); Norma Adams (1989), incorporado en su tesis de Licenciatura; Pablo Sandoval (2001) que sinteriza los aportes de la antropología en este campo y las nuevas temáticas vinculadas a la cultura urbana ${ }^{1}$; y los trabajos complementarios de Julio Calderón Cockburn (1990, 2014) que de manera más amplia que los anteriores, resumen bien la producción intelectual en el terreno urbano en el Perú. Todos estos trabajos significan buenas puertas de entrada al tema.

Existen pocas coincidencias entre los trabajos. Algunos balances realizados por los sociólogos son enfáticos en señalar que la antropología se encuentra casi ausente en el abordaje de esta temática, sin embargo, en sus referencias bibliográficas tampoco mencionan producciones universitarias, lo que sugiere la necesidad de elaborar un análisis inmediato que permita conocer las diferentes tendencias y características de estas producciones y así aportar a una comprensión más amplia del lugar de las ciencias sociales en el abordaje de la problemática urbana en el Perú.

\section{Lo urbano desde la antropología en San Marcos}

\subsection{Cultura andina en la ciudad y aculturación (1950 y 1960)}

El inicio de la antropología urbana en el Perú tiene conexión casi directa con los inicios de la etnografía en San Marcos, debido a que los iniciadores en el abordaje de la problemática urbana desde la antropología eran profesores del entonces Instituto de Etnología de la Faculta de Letras, sobre todo Valcárcel y Matos Mar; eran tiempos en que la antropología en el Perú era casi sinónimo de antropología en San Marcos.

Los primeros estudios de Matos se remontan a la década del cincuenta y cuyos resultados fueron publicados recién en la segunda parte de la década del sesenta (1967). Los estudios urbanos de la antropología en San Marcos no son tan recientes como se piensa cotidianamente. Las producciones de monográficas y etnográficas grafican que el tema urbano no ha sido ajeno a la antropología desarrollada en San Marcos.

Las preocupaciones centrales de las tesis de las primeras décadas giran en torno al tema de migración del campo a la ciudad, aculturación y formación de barriadas, con un alto contenido valorativo, sobre todo acerca del proceso de migración como de la constitución y estructura de las barriadas. Los estudios pioneros sobre antropología en la ciudad no tenían temas específicos de desarrollo (Meneses 1998) sino tendían a ser una etnografía "general" de un territorio específico.

La primera tesis pertenece a E. Mildred Merino de Zela de 1958. Es un estudio sobre la formación de la barriada de El Cerro San Cosme, para optar el grado de Bachiller. Tiene una evidente influencia indigenista y culturalista. El trabajo de $\mathrm{Merino}^{2}$ no tienen un tema específico, estudia la barriada de El Cerro San Cosme, su

2 Mildred Merino (1958): "Permítaseme dejar constancia de mi agradecimiento a las constantes y ejemplares observaciones de mi distinguido maestro y amigo Dr. Jorge C. Muelle; al Dr. Luis E. Valcárcel y Br. José Matos Mar que tanta facilidades me proporcionaron para el mejor tratado de mi tesis, especialmente del Censo realizado en 1949; al Dr. Félix Denegri Luna e Ing. Fernando Braschi, del Fondo Nacional de Salud y Bienestar Social, quienes me facilitaron la consulta de algunos documentos, y al Dr. Jorge Basadre, quien como Ministro de Educación, muy generoso y comprensivamente me dio la oportunidad de abreviar la terminación de mi tesis tan asediada por la falta de tiempo, eterno problema del estudiante que trabaja. Así la "buena suerte" del Cerro San Cosme en hacer amigos y oportunidades favorables, me alcanzaron.

A mi buen amigo Dr. Mario C. Vásquez y al Dr. Jean Vellard, mi gratitud por su gruía, su amistosa comprensión y estímulo. Al Dr. José María Arguedas mi agradecimiento...” (p. 5).
Respecto a la cultura urbana, véase también: para el caso de Lima, Salazar (2016) y Damonte (1994); para los casos de Lima, Santiago y Buenos Aires, Mathews (2016) y para Buenos Aires, Gorelik (2013). 
formación y cómo ha llegado a ser así. "Quiénes lo forman, de dónde provienen, qué fuerzas han promovido u obstruido el desarrollo del poblado; hemos tratado de seguir sus efectos en la vida de los hombres y en todo el sistema moviente. Gran ambición nuestra sería que esta monografía contribuyera como elemento de juicio para el estudio de quienes se aboquen a encontrar medios para dirigir aquellas fuerzas" (Merino 1958, 4)

Considera que el surgimiento de las barriadas ha generado que Lima se convierta en un problema social. Esto tiene correspondencia con las concepciones sobre las barriadas por esas décadas, 40 y 50, sobre las barriadas, consideradas como "problema" y "cáncer social”, que llevó al surgimiento de ideas contrapuestas que más bien concibieron las barriadas en el sentido más positivo (Calderón y Maquet 1990). El Arquitecto Peruano, liderado por el arquitecto Fernando Belaunde Terry, propugnaba la idea de que las barriadas eran un problema social debido a la migración. La propuesta sobre la ciudad ${ }^{3}$ de EAP giraba que todo este problema era la falta de vivienda y, por consiguiente, había que construir más viviendas en provincias para evitar la migración de provincianos a la ciudad. Ante esta lógica negativa de las barriadas, los antropólogos ingresaron a discutir y vieron que las barriadas tenían características importantes de colectividad y representaban una nueva forma de producir la ciudad.

Describe las características geográficas y sociales de la barriada San Cosme y la compara con las comunidades andinas, pero se preocupa también por la salud mental de los habitantes de esta forma de organización social urbana. Aquellas condiciones de trabajo fácil y habitación precaria y barata, generarían la vagancia y la delincuencia:

Lima, en sus actuales condiciones de proliferación de Barriadas, se ha convertido en problema social; es un punto de atracción de determinado tipo de provincianos, hombres, jóvenes y adolescentes, por su oportunidad de trabajo fácil aunque con ganancias mínimas (vendedor ambulante), y habitación barata aunque muy precaria (choza alquilada). Este tipo de poblador no es el original de San Cosme. Esas condiciones fomentan a la vez la vagancia y la delincuencia, y ambos podrán ser detenidas de inmediato mediante una Ley Nacional de Barriadas, cuyo Proyecto como "Plan Nacional de Saneamiento y Legalización de Barrios Marginales" ha sido presentado hace muchos meses al Senado (p. 201).

3 Sobre la historia del urbanismo, véase Chueca (2016).
La cita evidencia un juicio de valor sobre las barriadas, las condiciones de su existencia y sus efectos en el comportamiento de los sujetos. A esto suma la posición política de cambiar el escenario, pues, por esos ańos las barriadas no tenían reconocimiento del Estado, lo que se lograría recién en 1961.

Resumamos algunas conclusiones y propuestas centrales de esta primera tesis:

a) El traslado del mercado sirvió de construcción de vivienda para el personal (los placeros), lo que originó posteriormente la formación de la Barriada.

b) La predominancia de migrantes desde la década del 40 hacia Lima de Ayacucho, Junín, La Libertad, Ancash, Huancavelica y sierra de Lima.

c) Similitud con las poblaciones andinas de muchos elementos de su cultura material, de su cultura espiritual, su organización comunitaria y el alto grado de integración comunitario.

d) El traslado de los males del centro a la periferia de la metrópoli debido a la falta de una solución integral y una demolición descontrolada de callejones inmuebles.

e) La influencia del trabajo comunitario de los sancosminos, profesionales y candidatos políticos en el progreso cultural y material del pueblo.

f) Factores negativos para la población: la política, los arrendatarios y la negociación (el capital).

La segunda tesis es de J. Walter Quinteros Salazar, presentada en 1965, donde analizó las características de la población obrera del fundo y otras instalaciones de la Universidad Agraria, en La Molina. A diferencia de Merino, este autor plantea las diferencias entre los grupos de migrantes: migrantes de primera y segunda etapa. Busca explicar las formas de producción de barriadas, dónde habitaron los primeros migrantes y sus contradicciones en el proceso de aculturación.

Para Quinteros, en término de geografía espacial, los primeros migrantes se establecieron en los callejones y corralones de los barrios tradicionales de Lima; mientras que los grupos posteriores constituyeron las barriadas. Todos estos grupos se caracterizan por una existencia netamente urbana, de ciudad. Pero alude la existencia de otros grupos de migrantes indígenas de esta segunda etapa que residían en las entonces haciendas que rodeaban Lima y que estaban incorporados a la agricultura o a la ganadería. Las diferencias más resaltantes entre ambos grupos de migrantes estarían en la ocupación y el lugar de residencia. En esta investi- 
gación resalta la forma cómo los migrantes sufren procesos de aculturación cambiando sus anteriores estilos de vida por una existencia totalmente urbana. La aculturación, siguiendo a Aguirre Beltrán, es comprendida como "el proceso de cambio que emerge del contacto de grupos que participan de culturas distintas. Se caracteriza por el desarrollo continuado de un conflicto de fuerzas, entre formas de vida de sentido opuesto, que tienden a su total identificación y se manifiesta, objetivamente, en su existencia a niveles variados de contradicción” (Quinteros 1965, 28).

En términos metódicos, estas primeras tesis se caracterizan por su amplio contenido descriptivo, tanto del escenario como de las características de las poblaciones que interactúan, y compara con los modos de existencia de sus pueblos origen o cambiando sus estilos de vida originales para adquirir un estilo de vida propiamente urbana.

En la actualidad, como señalaremos más adelante, la categoría "aculturación" ha sido dejada de lado en los análisis antropológicos y sociales, indudablemente, tampoco se ha reflexionado sobre las implicaciones de este olvido.

\subsection{Dependencia, marginalidad y formas de relación campo-ciudad (década de 1970)}

Por estos años resaltan las tesis de Guillermo Gutiérrez (1974), María Ysabel Tapia Takey (1976), Sonia Becerra Flores (1977), Alejandro Salomón Zugbi (1972), y Teófilo Altamirano (1977), con propósitos propios de explicar la realidad desde ciertas perspectivas teóricas casi definidas, tales como la dependencia, dialéctica materialista, marginalidad e histórico-estructural. Los estudios vinculan de manera clara las problemáticas locales y regionales con las nacionales.

Tapia Takey cuestiona el estudio solo del área metropolitana de Lima y Callao y la ausencia de investigaciones sobre el proceso de urbanización en otras regiones el país: "Es en virtud de esta necesidad que nosotros decidimos analizar el proceso de urbanización de una ciudad serrana como Huancayo, considerada precisamente como la más importante de la sierra central, y por ende, en el centro hegemónico del valle, a lo que se suma, el ser considerada como la tercera ciudad de mayor importancia en el movimiento migratorio después de Arequipa y Cuzco, con el acrecentamiento de problemas, tales como marginalidad y tugurización, con situaciones comunes tales como: desempleo, sub-empleo, delincuencia, mendicidad, etc." (Tapia 1976, Introducción). Analiza el proceso histórico de la región y vincula a su desarrollo económico y social posterior. Señala aquí el papel que desempeña la ciudad de Huancayo, poniendo especial atención en los factores que la llevaron a constituirse en el centro hegemónico del valle; busca explicar la interrelación entre el crecimiento económico, el proceso de migración y la difusión de un proceso de urbanización rural que se desarrolla entre las décadas del 40 al 60; así como el vínculo entre el medio físico y la estratificación social de la urbe, así como el proceso de industrialización de la ciudad, partiendo del análisis de las causas que la originan así como de la repercusión de este proceso no solo a nivel local, sino también a nivel regional.

Las preguntas que formuló son las siguientes: ¿De qué manera el establecimiento de una economía de enclave, como fue el implantado por la Cerro de Pasco, afecta a la estructura económico-social tanto de los sectores urbanos, así como de los sectores rurales? ¿A partir de qué fecha y quiénes conforman la élite mercantil que se consolida en Huancayo, cuyas actividades influyen decisivamente en el desarrollo económico del área? Los cambios económicos producidos en el contexto nacional entre las décadas del 40 al 60, ¿de qué manera afectan a los centros de poder regional? ¿A qué factores se debe la existencia de un proletariado dual no solo en las zonas mineras, sino aún en centros urbanos como Huancayo?

El desarrollo de las hipótesis de Tapia están asociadas a las ideas de urbanización dependiente de Manuel Castells, de quien rescata su planteamiento sobre el proceso evolutivo del espacio latinoamericano con signos de las relaciones de dominación y dependencia de cada uno de los países de América Latina, configurándose con tres formas de dominación y relaciones de dependencia: dominación colonial, dominación capitalista-comercial y dominación imperialista (industrial y financiera). Adquieren mayor importancia las nuevas formas de dominación económica imperialista después de la segunda mundial.

Tapia rescata la idea del proletariado dual, relaciones de poder regionales, relaciones de dominación y de dependencia, cambios culturales y económicos, inmigración y relaciones estrechas entre ciudad y campo a través de relaciones comerciales directas. Se opera una lectura de la conversión de Huancayo en una hegemonía del valle del Mantaro, que significó el reemplazo 
en este papel a Jauja y los cambios en la dinámica de la sierra central. Sostiene varias hipótesis:

La primera plantea que a nivel de la población rural el trabajo en las minas, a partir de la instalación de la minera Cerro de Pasco, provoca alteraciones importantes en el valle. Permite el surgimiento de un proletariado dual y hasta de triple actividad, pero que nunca dejan de ser campesinos debido a que están ligados a la tierra por la propiedad de sus parcelas. Mientras que a nivel del sector urbano, se presenta la quiebra del poder de la élite tradicional y el surgimiento de nuevos grupos ligados fundamentalmente al comercio.

Los cambios que produjo la construcción de la vía ferroviaria en 1903 y la carretera central en 1920 aparecen como trascendentales, debido a que acentuaron la migración tanto de extranjeros como de costeños, una élite cuyo poderío estaba basado principalmente en sus conexiones con las casas importadoras de Lima y las compañías mineras establecidas en la zona.

Antes de la construcción del Ferrocarril Central, es decir, hacia 1900, el valle del Mantaro no difería de los otros valles interandinos del sur. Se distinguían tres estratos sociales: indios, mestizos y blancos, diferenciados por la conducta, las costumbres, la vestimenta y la lengua; de modo que entre la cultura de los indios y "blancos" existía una diferencia sustancial, pero, y esto es importante de hacer notar estas diferencias se dan tan solo en el aspecto cultural, no así en lo económico ni en lo social. No se estableció entre blancos, mestizos e indios, relaciones de dominación y servidumbre derivadas como el pongaje, el colonazgo y el yanaconaje, o sea, relaciones de dominación y discriminación basadas en su diferente situación socioeconómica.

Pero el factor que aceleró el ritmo de las transformaciones económicas y sociales de todo el valle del Mantaro, fue la llegada de la línea del Ferrocarril Central a Huancayo en 1908. El ferrocarril significa para algunos pueblos como por ejemplo Sicaya; la muerte del arrieraje, la emigración, el encarecimiento progresivo de la vida, que hacía cada vez menos posible el trabajo comunitario en las tierras de la Iglesia y la dependencia exclusiva de la agricultura (pp. 24-25).

Resalta, asimismo, la importancia del ferrocarril en los cambios en la economía de mercado a nivel regional y sus implicaciones:
A nivel regional, el ferrocarril significa también la intensificación de las actividades comerciales, ya que introduce cada vez más a los campesinos dentro de una economía de mercado, produciendo al mismo tiempo, la eliminación de progresiva del quechua, tomando imprescindible el castellano para las actividades comerciales...Es a partir de la llegada del ferrocarril que se acentúa la inmigración de extranjeros y el intercambio con la costa, dando cambios en la estructura económica de Jauja (que era todavía el centro dominante), al punto de permitir a nuevos grupos el monopolio comercial... (pp. 25-26).

Y a partir de la década del 50 en que el país entra en una nueva fase de su economía como resultado de la política de inversiones extranjeras se impulsa el crecimiento urbano mediante fuertes oleadas migratorias que se dan como efecto de la relación más estrecha entre ciudad y campo, a través de interrelaciones comerciales directas. Tapia conecta los cambios regionales con sucesos nacionales, sintetiza de ese modo los factores que originaron un mayor dinamismo y una nueva configuración de Huancayo.

Bajo el impulso de su gran actividad comercial, originados por la llegada del ferrocarril, la culminación de la carretera central, así como su feria dominical, Huancayo adquiere todas las características de un gran centro poblado, siendo considerado como el más importante de la región central, que irradia su influencia sobre toda la zona. A estos factores se suma el traslado de la capital del Departamento de Junín de Cerro de Pasco a Huancayo en 1,931; acerca de las causas que determinaron su traslado a Huancayo y no a Jauja se podría aducir una motivación política ya que los pueblos y comunidades de la parte del valle eran partidarios de Sánchez Cerro quien había derogado la Ley Vial, que constituía un elemento de explotación sobre la masa indígena. Por otro lado, al ser Jauja el principal centro leguiísta del valle, el Prefecto, hombre de confianza de Sánchez Cerro, optó por Huancayo (p. 36).

Una última hipótesis es que para esa época en la ciudad de Huancayo existía una gran cantidad de pequeñas empresas que operaban en forma clandestina, no sujetas a obligaciones tributarias, con muy pocos trabajadores generalmente unidos por relaciones de parentesco, quienes contrataban trabajadores asalariados eventuales. La autora distingue, por consiguiente, entre actividades comerciales formales e informales. Sin embargo, cuando Tapia realizó su investigación, en la 
década del setenta, Huancayo se encontraba en un proceso de desindustrialización y el estancamiento de su sector comercial.

En general, como se ha señalado más arriba, este trabajo continúa la línea de reflexión seguida por Manuel Castells. Fue asesorada, además, por Luis Millones que por entonces venía trabajando temáticas vinculadas a la marginalidad urbana.

Una segunda tesis en esta década del setenta corresponde a Teófilo Altamirano (1977) que investiga las identidades étnicas y la formación de asociaciones regionales en Lima Metropolitana, para su doctorado en Antropología; su finalidad es analizar los desarrollos regionales y locales estrechamente vinculados al desarrollo urbano de Lima.

Desde la perspectiva histórico-estructural, describe las causas externas en función a la influencia que ejerce el contexto urbano hacia las regiones y las localidades. Analiza también las causas internas en función a su desarrollo dependiente de las causas externas, además considerando su relativa autonomía al que se deriva de una constante utilización de sus propios patrones de organización social.

Altamirano ${ }^{4}$ estudia dos regiones distintas: el Valle del Mantaro (Junín) y el Valle del Pampas (Apurímac), teniendo como objetivo central analizar si las asociaciones regionales de los migrantes en Lima "se organizan como consecuencia de las identidades étnico-regionales; si esto es cierto, en qué medida los valores urbanos condicionan la formación y desarrollo de las asociaciones. La pregunta condiciona para que el estudio concentre sus objetivos a considerar con mayor énfasis las relaciones sociales del migrante fuera del contexto de las relaciones de trabajo al que necesariamente está vinculado; vale decir se enfatiza sus relaciones familiares; con migrantes de sus lugares de origen ("paisanos"); con otros migrantes de la misma región y con otros que muestran relativamente los mismos rasgos económicos, sociales y culturales...” (Altamirano 1977, 3-4).

Para el autor, las relaciones de parentesco juegan un rol importante en el proceso de formación de las asociaciones. El mayor vínculo parental permitiría mayor posibilidad de formar una institución. Descubrió que las instituciones en Lima están formadas en base

4 "El presente estudio es la continuación de dos investigaciones en las regiones citadas anteriormente, los que realicé en distintos períodos, en 1969-70 en el valle del Pampas y en 1971-72 en el Valle del Mantaro". (p. 10). al vínculo directo con el barrio de origen, lo que le llevó a pensar que hay una relativa reproducción de la organización social con las limitaciones que conlleva al estar en un contexto urbano. Es otros términos, las instituciones surgen frente a una problemática en el escenario urbano y adquiere conexión con los lugares de origen. Los miembros de las instituciones de cada barrio son parientes en primero, segundo y tercer grado de los miembros de las instituciones similares en Lima. En el caso que estos no sean parientes, pues, resultarían pertenecer al mismo barrio. Hace uso del método de las redes sociales para el estudio de caso, además del análisis situacional y estudio intensivo a nivel institucional, pero reconoce su limitada aplicación debido a que no han desarrollado mayormente esos campos.

Resumamos a continuación algunos de los resultados de Altamirano:

El primero es que el establecimiento y las mejoras de las vías de comunicación, la difusión continua de los medios de comunicación social (radio, TV y los periódicos, etc.) y los programas de desarrollo rural, de manera especial la educación formal, han motivado para que a partir de la década del cincuenta el proceso migratorio, sobre todo hacia los centros urbanos, los centros mineros, hacia las haciendas agro-industriales de la costa y hacia los centros de colonos de la selva. Plantea las condiciones y características de las fases de la migración y la forma cómo fue cambiando para optar otras dinámicas.

La migración urbano-rural en sus primeras fases fue muy selectivo; eran los componentes de las burguesías provincianas que empezaron a poblar Lima; estos grupos no perdieron sus vinculaciones con sus regiones de origen, porque allí estaban sus bases económicas y sociales y además de tenerlo a nivel nacional para su participación en el Estado, como representantes parlamentarios o vinculados a los grupos de poder económico urbano. Las relaciones con sus regiones de origen; además la identificación de intereses económicos y sociales comunes entre estos migrantes y finalmente el deseo de mantener las costumbres de sus respectivas regiones motivaron la formación de los clubes departamentales y provinciales. (...) (pp.100-101).

La segunda conclusión es que en la medida en que el proceso de migración fue incorporando a vastos sectores campesinos particularmente de la sierra central y sur, las asociaciones regionales fueron por esos tiem- 
pos mantuvieron un crecimiento de sus miembros; y al mismo tiempo se crearon otros. El flujo migratorio entre Lima con otras regiones permitió la consolidación de las asociaciones, lo que generó además la emergencia de instituciones "filiales" en las comunidadesdistrito, estableciéndose relaciones de dependencia de estas respecto de las existentes en Lima; así como el surgimiento de relaciones de intercambios económicos, sociales, religiosos y políticos, de manera especial con los barrios de origen con la organización comunal y/o distrital.

Concluye que el flujo de estos intercambios genera una acelerada "urbanización" de las estructuras tradicionales; convirtiéndose así las instituciones en agentes de cambio para el proceso de innovación y modernización especialmente en los sectores rurales de las regiones. Pero en algunos casos la organización comunal se ha visto reforzada con las relaciones interinstitucionales, constituyéndose las de los migrantes en portavoces de las tareas internas de las comunidades ante los organismos estatales, sindicales y políticos. Las organizaciones regionales de los residentes en Lima constituirían formas organizadas de representación de los sectores más bajos de la estratificación social y económica urbana.

Alejandro Salomón Zugbi presentó su tesis en 1972 para optar el grado de Bachiller en Antropología. Estudia la formación del pueblo joven "Año Nuevo", en el distrito de Comas, después de la Ley $13517^{5}$, del 14 de febrero de 1961, durante el gobierno de Manuel Prado, que en su artículo $2^{\circ}$ prohibía la formación de "barrios marginales". Busca describir y explicar la realidad de lo cambios ocurridos en este pueblo joven a partir de la intervención del Estado y la actuación de sus dependencias para el mejoramiento de su establecimiento, convirtiéndolo en un modelo urbanizable y agregarlo a la comunidad local.

Salomón desarrolla su análisis desde la perspectiva de la marginalidad; plantea la poca posibilidad de los pobladores para superar su condición de subdesarrollados. Su preocupación se centra en el problema de la vivienda, tendencia casi generalizada desde mediados del siglo XX. El pueblo joven ${ }^{6}$ mencionado en 1967

5 Salomón cita el contenido de esta Ley: "Los que se formaron o intentasen formarse no obstante esta prohibición — dice el textoquedan excluidos de los beneficios que esta Ley concede solo para los barrios marginales existentes" (Salomón 1972, Introducción).

6 "La nominación de "pueblos jóvenes" fue dada por el actual gobierno revolucionario de la Fuerza Armada, porque consideraba la denominación de Barrios Marginales o Barriadas demasiada peyorativas y humillantes, por lo que mediante el Decreto Supremo 105-68-FO, proscribió el nombre de barriada" (Introducción). y terminó amparándose, como menciona el autor, en otras disposiciones de Ley. Asume que el problema de la vivienda y la formación de barriadas son producto del crecimiento demográfico, del bajo nivel de vida en el país y la migración hacia la capital.

Analiza también la actitud de los individuos, quienes expresan rasgos psicosociales negativos de la vida, tales como malhumor, pesimismo, resignación y fatalismo, lo que devendría en una introversión y una fuerte desconfianza en relación a los demás. Estas actitudes y expectativas deformes sobre la vida se producirían debido a la condición de marginalidad que tienen en una sociedad estratificada económicamente. Pero estas actitudes podrían ser desplazadas con una labor de concientización y la búsqueda colectiva de los medios para superar estas condiciones de subdesarrollo.

Salomón confía en que los programas de gobierno logren dejar de lado la condición de marginados de los pobladores. Esto se asemeja a ideas de la marginalidad funcionalista explicada por Meneses (1998), es decir, la promoción de "programas de desarrollo comunales" terminan cumpliendo un rol fundamental para la incorporación de los marginados al desarrollo. Hay un dualismo entre desarrollados y subdesarrollados, con cierto tipo de actitudes descritas en el caso de los segundos debido a las condiciones de dominación en la que se encuentran:

Casi todos los pobladores, se sienten y convierten en objetivo de una acción planificada y tendiente a promover el desarrollo integral de su condición humana pero, el analfabetismo, la promiscuidad y la desnutrición se ven reflejadas en su actitud y en los conceptos errados que tienen de la vida, nos han mostrado rasgos psicosociales evidentemente negativos que son notorios al estarse continuamente malhumorados, pesimistas, resignados y casi fatalistas, que los condicionan a una introversión y una fuerte desconfianza para con los demás (Salomón 1972, 73).

En 1974, Guillermo Gutiérrez presenta una tesis en donde busca analizar la realidad interna de la barriada El Porvenir ${ }^{7}$, en Trujillo, La Libertad. Estudia sus contradicciones y aspectos más resaltantes, desde una

"La Barriada El Porvenir, es el conglomerado social más antiguo, que ostenta la capital del Departamento de La Libertad: Sus orígenes se precisan en 1934, cuando fueron a vivir cuatro familias que constituyeron, en aquellos arenales desérticos, el primer enraizamiento social, que sirvió de pauta para el camino hacia la plasmación de lo que hoy en día es El Porvenir. (...)” (p. 41). 
perspectiva marxista, y toma como método la dialéctica materialista, lo que le lleva a considerar la unión entre la teoría y la práctica. Considera que las barriadas del Perú son producto de la contradicción entre la ciudad y el campo, consecuencia del desarrollo interno de la estructura económica al capitalismo dependiente.

La barriada connota, para el autor, una multiplicidad de contradicciones recíprocas de carácter económico, político, social y cultural, por estar dentro de un régimen semifeudal, neocolonial, en transición al capitalismo dependiente. Estas exigencias de enfoque, devendrían como resultado de la dialecticidad de su realidad. Entre los factores que originan las barriadas incluye a los movimientos migratorios internos, el subdesarrollo económico, el crecimiento demográfico, la escasez de viviendas, los bajos ingresos de la población, la desocupación y el centralismo político.

Hace también una lectura de la composición de clases sociales en El Porvenir y realiza la siguiente clasificación: pequeńa burguesía (con sus dos sectores: media y baja), semiproletariado, proletariado y lumpemproletariado. ¿Quiénes componen cada una de estas clases sociales?

La burguesía media estaría compuesta por pequeños comerciantes, artesanos y pequeñas industrias; mientras que la pequeña burguesía (baja) por profesores, tenderos, estudiantes universitario, profesionales, comerciantes minoristas, artesanos dueños de carpinterías, zapatería panaderías, choferes y por empleados públicos.

El semiproletariado estaría definido por pequeños artesanos, dependientes, vendedores ambulantes, canillitas, empleadas domésticas, modistas y agricultores. En tanto, el proletariado, el sector más numerosos y progresista, estaría constituido por trabajadores de factorías y fábricas de Trujillo, donde venden su fuerza de trabajo. Además, señala que el proletariado es la clase social que tiene la misión histórica de cambiar el sistema económico para instaurar una sociedad socialista.

Y en el lumpemproletariado están los considerados como "desclasados" socialmente, vistos como el lastre social en la barriada El Porvenir. Se encuentran aquí los delincuentes, prostitutas e indigentes. Para el autor, estos sujetos tienen una tendencia destructiva y llevan una "vida parasitaria" y "perniciosa" (Gutiérrez 1974, 66).

Finalmente, entre sus conclusiones centrales podemos señalar: a) el proceso de la estructura económica a una capitalismo dependiente y el surgimiento de las barriadas como parte de ese proceso de transición y de la contradicción entre el campo y la ciudad, b) la relación directa entre el fenómeno social de la barriada y la problemática de la vivienda; ambas problemáticas se agudizan con la sociedad capitalista, y sería superado con la construcción del socialismo; c) pobladores de las barriadas como posibles sujetos sociales políticos representantes de las luchas políticas e ideológicas frente a organismos de opresión; d) supresión de la migraciones del campo a la ciudad solo a partir de la eliminación de la contradicción que genera la sociedad de clases que promueve al mismo tiempo el centralismo político y cultural respecto a otros pueblos del Perú; y d) contradicciones entre el mismo pueblo a pesar de que los generadores de estos fenómenos son externos.

La tesis de Gutiérrez tiene un trasfondo ideológico y político. Los resultados están seguidos de juicios y propuestas políticas para cambiar la sociedad dominante por otra nueva: el socialismo, sociedad que, según el autor, eliminaría la problemática de la vivienda, la contradicción entre ciudad y campo y, por consiguiente, el surgimiento de las barriadas. En el tema de la vivienda, asume la tesis de Federico Engels.

La tesis de Sonia Becerra Flores, presentada en 1977 para optar el grado de Bachiller, estudia etnográficamente al pueblo joven Medalla Milagrosa de Magdalena del Mar. Busca entender su situación, las causas de su origen, así como las perspectivas y aspiraciones de sus miembros.

Esta tesis contiene ideas como migración campociudad, barrios marginales, urbanización acelerada de grandes ciudades, dependencia e individualización de objetivos. Para la autora, la migración y la constitución de barrios marginales son fenómenos socio-económicos de carácter estructural, producto de factores como la dependencia externa (internacional) y la dependencia interna (nacional), que al mismo tiempo tienen una relación estrecha. Nuevamente, esta tesis vincula el surgimiento de las barriadas con una situación dependiente de la sociedad nacional frente a agentes externos y la dependencia interna.

Desde la perspectiva de la marginalidad, Becerra desarrolla las hipótesis partiendo de una explicación de la relación campo-ciudad a través del proceso migratorio que trae como consecuencia la densificación demográfica y la reducción de del espacio disponibles en áreas urbanas, generando el surgimiento de las barriadas. Es una visión crítica de las barriadas y del proceso migratorio, busca explicar y problematizar los efectos que tiene. La migración estaría además asociada a las 
expectativas y aspiraciones de los sujetos por mejorar sus niveles de vida.

Encuentra que las aspiraciones de los pobladores del pueblo joven están desligadas del interés comunal, y a medida que alcanzan mayor estatus sus intereses son más individuales.

\subsection{Lazos y estrategias campesinas en el contexto urbano (década de 1980)}

En la década del ochenta existe una sola tesis y es la de Norma Adams Alegre (1989) ${ }^{8}$. Esta es una de las décadas de mayor producción intelectual sobre la problemática urbana en el Perú, pero en contraste, la situación es todo lo contrario en la producción etnográfica en San Marcos.

El interés central de Adams es explicar en qué grado algunas instituciones y formas de interacción provenientes del campo, como es el caso de la organización campesina, son trasladadas, reelaboradas y reinterpretadas en el contexto urbano para solucionar los problemas que enfrenta el migrante. Otro aspecto resaltante de su interés es explicar la migración como estrategia de grupos de campesinos para su adaptación a una nueva situación urbana. Así, aborda la importancia del origen campesino y la relación que tiene con el lugar de origen un grupo de migrantes en el proceso de urbanización de Lima.

Busca hacer una reevaluación del proceso migratoria y el proceso de urbanización, así como la diferenciación cultural y étnica, y la importancia de las relaciones de parentesco. Pero reconoce que la metodología no les permite desarrollar más que grupos cultural o étnicamente similares. Parte de la idea de que las instancias de agrupación étnica y familiar son mecanismos que organizan la vida de los migrantes, tienen una funcionalidad como un filtro de adaptación colectiva a la sociedad. A diferencia de Degregori, Lynch y Blondet (1986), no estudia grupos territorializados sino lazos de tipo étnico y familiar y su importancia en la vida de las personas. Es decir, el parentesco tiene importancia en la vida familiar así como en el manejo de la asociación formal.

8 "El estudio parte de un trabajo de campo que se inició bajo el auspicio del Proyecto Perú-Cangallo "Migración y Salud Mental" (La responsabilidad en la parte antropológica del proyecto estuvo bajo la dirección del Dr. Héctor Martínez) que culminó en el texto presentado a AMIDEP "Migración y Salud Mental en el Perú. Planteamientos, problemas y avances" Ms. 1983. H. Martínez, N. Adams y C. Peñafiel" (pp. 14-15).
Para la autora, existe una incidencia de la cultura del pueblo de origen en el devenir de migrantes individuales. Los lazos en base a las lealtades interpersonales derivadas de los patrones culturales del pueblo (familia extensa, parentesco espiritual o ceremonial, lazos comunales, cofradías, grupos religiosos, etc.) permiten elaborar estrategias comunes de inserción en la ciudad. Encuentra que los migrantes en parte mantienen una vinculación estrecha con sus pueblos que contribuye a preformar la imagen urbana en los potenciales migrantes.

La construcción de las redes de apoyo mutuo de los migrante serviría como un elemento de equilibrio para el desenvolvimiento en la ciudad, en la cual el asalariamiento simple, ya desde su inicio requeriría de condiciones especiales como de una reconsideración donde la reproducción requeriría de una multiplicidad de estrategias y donde la pertenencia a un grupo mayor podría servir de base para un tipo de crecimiento que necesite características de producción y de "intermediación precapitalistas”, que requeriría de vínculos como los que les ofrece la cultura campesina.

\subsection{Lima, adaptación de migrantes y elementos culturales (década de 1990)}

Esta etapa se caracteriza por el surgimiento de diversos temas pero uno de los aspectos más resaltantes es el inicio del abordaje de la globalización. Son investigaciones con temas más específicos que incluyen la música, economía, la globalización, localidad, cultura y poder. Algunas investigaciones de este periodo tienden a ser comparativas. En conjunto, se trata también, en términos teóricos y metodológicos, de producciones más difusas y eclécticas, toman múltiples autores para construir sus argumentos. En la década del noventa existen solo tres trabajos, el de Ladislao Landa (1992), el de Gabriela González Carbajal (1994) y el de Mario Vallejos Burgos (1996). Dos de ellos trabajan de manera directa la adaptación del migrante.

Landa' (1992), además de buscar entender la realidad y características de los diferentes movimientos

9 "(...) hemos optado en utilizar los diferentes discos para nuestro estudio. Y hemos seleccionado un universo de 73 Long Plays de los Archivos del Programa "Tierra Fecunda" del CEPES (Centro Peruano de Estudios Sociales), los cuales hemos fichado y escuchado, escogiendo lo más representativo para determinar en base a ellos las características de las corrientes musicales. (...)". (Landa, 1992: 10). "Otra de nuestras fuentes de información han sido las entrevistas a diferentes personas con el fin de testimoniar sus experiencias. En este caso hemos dividido en dos tipos de informantes, a los cuales denominamos: testigos y creadores". (Landa 1992, 10). 
musicales de ese entonces, tiene el propósito de estudiar de manera detallada los modos de adaptación de los migrantes y sus cambios en las ciudades a través de la música, de tal modo que llega a estudiar los cambios de la estructura o contenido de la música andina en la urbe metropolitana. Busca explicar el fenómeno musical de origen provinciano y su desarrollo en Lima desde las primeras grabaciones en el Perú (1949) hasta la crisis de la disquera (1989), haciendo énfasis en el huaino.

Si Mangin y Doughty estudiaron el proceso de migración, la formación de las asociaciones regionales y el proceso de migración desde el lugar de origen de los migrantes y Matos Mar un reconstrucción desde la ciudad y el proceso de incorporación de sus elementos culturales andinos, Landa estudia las situación de la música andina en espacios citadinos (el huaino) y los cambios que ha experimentado; es decir, cómo se reflejan los cambios sociales en el arte como la música. Comprende que es una música urbana de origen andino y no una música propiamente andina, ya que tiene modificaciones y componentes que se alejan de su sentido inicial pero buscan seguir representándose como música nacional, se refiere con especial énfasis a los cambios de contenido del huaino.

Para el autor, el mercado reestructura y modela la producción artística que implica la producción (composición), distribución (difusión) y consumo de las canciones, y deben pensarse en los planos de realización de la mercancía. Al mismo tiempo, menciona que los cambios a nivel social están también reflejados en la música.

Algunas conclusiones de Landa: 1) Las teorías que ayuden a explicar la realidad de los cambios en las ciudades a nivel de la superestructura aún no son muy coherentes ni acabadas, 2) los grupos musicales que han salido de los esquemas básicos del huaino peruano se alejan cada vez más de esta matriz peruana (el huaino criollo, música latinoamericana, la chicha) para crear nuevas formas pero continúan afirmando su representación a la música nacional, 3) el huaino, música creada en el Perú, a pesar de la creación de esas nuevas formas, aún no perdido su esencia que lo ha mantenido como música representativa de nuestro país y 4) el sector marginal (informalidad) es el que domina este el "negocio" de la música, debido a la existencia de un reducido interés del Estado por mantener este importante componente de la cultura.

La tesis de Mario Vallejos Burgos (1996), por su parte, estudia la adaptación de los migrantes del distrito de Chiara (provincia de Andahuaylas, Apurímac) en Lima Metropolitana, de manera específica en el pueblo joven Manuel Dulanto, de la Provincia Constitucional del Callao. Busca estudiar "de qué manera un proceso de migración rural/urbano y la consecuente adaptación de los migrantes a la realidad cultural de las ciudades, trae modificaciones con los elementos culturales materiales e inmateriales que los migrantes son portadores desde sus comunidades de origen" (p. 29).

El contenido de esta tesis está marcado por ideas que incluyen la migración, adaptación social, valores tradicionales, solidaridad social, actividades mágicoreligiosas, control social, asimilación, organización tradicional, ayuda mutua y violencia. ¿Cuál es la relación que existe entre la migración y las demás variables? Esto es uno de los interrogantes que trasciende la tesis.

Parte del supuesto de la relación entre la adaptación social y valores tradicionales (como la solidaridad social) frente a la cultura urbana. Considera que la solidaridad es parte de la "ancestralidad" de las comunidades andinas.

Según Vallejos, el conocimiento de la cultura de origen de los migrantes podría ayudar a explicar conductas como normas de socialización, afán de logro, expectativas sociales, mecanismos de solidaridad de los migrantes entre sí y con sus lugares de origen. El proceso de inserción del migrante evoluciona desde la etapa inicial de adaptación para llegar posteriormente a la asimilación; esta última supone la fusión de culturas. El avance de la adaptación a la asimilación hace que suceda una modificación las prácticas que provienen de las comunidades de origen debido a la influencia de la cultura urbana pero sin perder su vigencia. Y de la interacción de los valores culturales provenientes de las comunidades de origen y la cultura urbana se produce una cultura mestiza, basada en valores comunales y citadinos, andinos y costeños, que tienden a fortalecerse en una nueva cultura que paulatinamente se impone en el Perú.

Otra tesis en la década del noventa es la de Gabriela Gonzales (1994). Estudia desde el uso de materiales arqueológicos y la etnohistoria la Lima Prehispánica: "El objetivo de estudio es una descripción de la evolución o desarrollo de los pueblos que antecedieron a la conquista española en lo que es ahora el departamento de Lima (Costa Central del Perú). Para abordar el tema nos vamos a referir a los centros culturales más importantes que se desarrollaron a lo largo y ancho de las 
siete provincias que conforman lo que es actualmente el departamento de Lima” (p. 2).

Considera que fuimos más que simples caseríos antes de la llegada de los españoles y que hubo un pasado histórico que duró más milenios de lo que se pensó y que lo que ha durado como historia hispánica y republicana. Los recursos del mar habrían permitido que en la costa se dieran las condiciones para el temprano sedentarismo de los primeros pobladores del litoral, lo que habría llevado al desarrollo y evolución de las sociedades arcaicas. Para la autora, los ríos jugaron un papel trascendental en la evolución tanto de los pueblos de la costa, como de la sierra, porque sirvieron para la formación de canales de riego, andenerías y otras obras hidráulicas. A partir de datos arqueológicos asume que la sierra de Lima tuvo su desarrollo bajo la influencia de tres corrientes: “a) los Huanchos (Centro-Este) en la parte baja de lo que hoy es la provincia de Huarochirí. b) Los Yautos (Sur-Este) que fueron los Checas y los Chacllas, en la parte alta del río Mala, llegando hasta Cocachacra y San Bartolomé. c) Los Atavillos (NorteEste) que abarcó la sierra de Chancay, Canta y Cajatambo" (p. 158).

Hace énfasis en que las culturas limeñas se encontraban en avanzado estado de desarrollo socio-político cuando llegaron los incas. Estaban organizadas en curacazgos o señoríos que controlaban más de un valle (Ychmay, por ejemplo, se extendió sobre la parte bajo de Lurín y Rímac). Este avance y desarrollo se habría reflejado por dos hechos importantes: a) La tenaz resistencia a ser invadidos y b) La conservación de formas organizativas y de logros infraestructurales por parte de los incas.

\subsection{Ciudad globalizada, hegemonia y relaciones de poder (2000-2012)}

A pesar de ser teóricamente eclécticas, las investigaciones de los inicios del siglo XXI son marcadas por ciertas tendencias resaltantes como el posestructuralismo, la sobremodernidad, los estudios culturales y los estudios subalternos o postcoloniales. En cuanto a temática de interés, predomina el estudio de migrantes pero ahora con temas más específicos en contraste a los estudios de las primeras décadas. Se analiza al migrante pero ya no solo su traslado desde el campo a la ciudad limeña sino de esta a otras metrópolis del mundo, por lo que la misma ciudad de Lima aparece como un espacio dinámico e interconectado: ciudad globalizada.
Las investigaciones contienen otro corpus lingüístico, más asociado a lo global, lo local, microfísica del poder, el lugar y el no lugar, identidades, heterogeneidad, la configuración de la poli o multicentricidad ${ }^{10}$ limeña, las dinámicas de los megamercados y los migrantes transnacionales. Reaparece la idea de lo marginal pero con contenidos que buscan discutir su relación con áreas consideradas hegemónicas.

La tesis con la que inicia la producción antropológica sobre lo urbano en el siglo XXI es la de Gloria Ríos Espinoza ${ }^{11}$ (2000). Investiga las visiones de la madre migrante relativo al cuidado de la salud del niño y su vínculo con medicina tradicional, en un espacio al que considera un área urbana marginal. Su interés está también en las ideas de los diferentes organismos respecto al cuidado de salud del niño, los procesos que intervienen en la toma de decisiones, la percepción de la población respecto a las acciones de salud y sus agentes, las situaciones adversas y favorables del cuidado de la salud del nińo, el papel que cumplen las redes sociales, la familia, las redes de parentesco, instituciones y organizaciones, así como el desarrollo de los procesos cognitivos de ser madre en la red social.

$\mathrm{Su}$ estudio es desde la perspectiva de la marginalidad de Larissa Lomnitz, sumado a la propuesta de Amartya Sen en cuanto a la propuesta de desarrollo desde los derechos humanos. Y para la definición de cultura, recurre a Clifford Geertz, es decir, la cultura como un concepto semiótico e inserto en una trama de significados. Se trata de una tesis sumamente ecléctica.

Asevera que la cosmovisión andina, las condiciones materiales de existencia y las redes sociales (expresadas en las relaciones de familias, parentesco y las instituciones), en las que se inserta la madre, juegan un rol

10 Arroyo (2014, 2015) denomina a Lima como una metrópoli policéntrica, mientras que Jacinto (2014) prefiere definirla como multicéntrica.

11 "Mi inquietud para definir el tema de la tesis surgió al egresar de la Escuela de Antropología, y atraída por la medicina tradicional, decido hacer trabajo de campo sobre dicho tema en un área urbano marginal de Lima elevada concentración de población migrante, y soy invitada por el coordinador del Proyecto Red Integrada de Salud de Salud (REDISS) de la Universidad Peruana Cayetano Heredia en convenio con la Universidad Laval de Quebec a participar como miembro del equipo.

Dicho proyecto se desarrollaría en el distrito de Independencia en el año de 1992 (permanecería trabajando en el mencionado proyecto hasta 1996). Durante todo ese lapso de tiempo, permanecí trabajando en la comunidad, con las organizaciones de base y los agentes de salud, y es en él donde se da el proceso de la observación participación del presente estudio. Las entrevistas a las madres de los casos fueron realizadas en el año 1994, y los grupos focales con las promotoras de salud se aplicaron dos en 1994 y uno en 1995” (Ríos 2000). 
importante en las prácticas de salud de las madres migrantes en relación a los cuidados de la salud del niño. Estos tres aspectos, sin embargo, no tendrían el mismo peso ni serían homogéneos.

En el año 2001, Javier Ávila Molero presentó sus tesis que investiga el tema de globalización y migración. Su preocupación se centra en la relación de los migrantes de la comunidad San Antonio de Cocha en Lima y en los Estados Unidos. Analiza las tensiones regionales, étnicas, de clase, generacionales y de tipo que la globalización viene produciendo entre los migrantes de San Antonio de Cocha (y sus hijos) en Lima y los Estados Unidos así como las que viene produciendo entre los que se quedaron o decidieron regresar a la comunidad. Estudia a la organización de los migrantes en Lima "Centro Unión Hijos de San Antonio de Cocha" y en la ciudad de Paterson (New Jersey, Estados Unidos) el "Club Social San Antonio de Cocha-Ayacucho". Formula las siguientes preguntas: ¿Cuál es rol que entre los migrantes de esta comunidad en Lima y los Estados Unidos vienen jugando las redes de parentesco y paisanazgo? ¿Por qué estos migrantes mantienen y refuerzan identidades locales en el medio del actual proceso de globalización? ¿Cuáles son las tensiones generacionales y de género que se manifiestan al interior al interior de sus familias? ¿Qué influencias tienen sobre su lugar de origen? ¿Cuáles son los cambios por los que viene atravesando la comunidad.

Ávila considera que la globalización y la creciente desterritorialización de las culturas e identidades genera entre algunos grupos de migrantes campesinos y sus hijos, descendientes de antepasados "indios", crecientes necesidades de (re) construcción de prácticas, identidades, discursos vinculados con su comunidad de origen, como parte de un repertorio de estrategias materiales y simbólicas que se agencian para enfrentar el desafío de la inclusión/exclusión en grandes ciudades del Perú y el extranjero. Se trataría de un fenómeno cultural que toma como fundamento un pasado "inventado" por las poblaciones migrantes en medio del proceso de globalización.

Esta recreación de símbolos, ritos y memorias campesinas en el imaginario de los migrantes urbanos, hace posible su uso en los procesos de reconstitución de identidades en espacios de la ciudad que inicialmente les resultaron extraños: el fenómeno se produce a través de diversos y complejos mecanismos de "hibridación" cultural, los cuales apuntan a reforzar discursos y referencias arraigadas en una experien- cia social y cultural original compartida por los migrantes. Si bien el fenómeno no es nuevo, cualquier antropólogo urbano más o menos atento puede apreciar como este se viene intensificando en pleno siglo XXI, en medio de los cambios y la vorágine del proceso de globalización (pp. 14-15).

Entre los principales resultados a que llega se encuentran: El primero se refiere a la relación entre "globalización" y "localización", es decir, la presencia de la paradoja sobre la existencia simultánea entre la "desterritorialización" y "reterritorialización" de identidades, fortaleciendo hábitos, identidades y la construcción de sentidos de comunidad local. Segundo, considera que la migración masiva de millones de campesinos convirtió al Perú de un rural en uno urbano, y que los migrantes tuvieron la ocasión de reinterpretar y reelaborar en la ciudad manifestaciones "invisibles" de su cultura, como las redes sociales de parentesco y paisanaje, que junto a una ética del trabajo campesina andina y otros saberes y habilidades rurales, forman parte de un capital social y cultural muy importante en las diversas estrategias económicas de sobrevivencia y empresariales desarrolladas en Lima. Tercero, la existencia creciente del tráfico de bienes materiales y simbólicos a través de estas redes, una creciente "fluidez" de identidades y ofertas para el consumo cultural que se incrementaría con la globalización.

Pero también nos demostró que esta "fluidez" de identidades y ofertas de consumo cultural dentro de la red tenía "compuestas" o "válvulas" que regulaban el tráfico dentro de ella. Y eran "compuestas" o "válvulas" estrechamente relacionadas con las variables "clásicas" de clase social, etnicidad, género y generación. Es decir, mientras que una persona está ubicada en la comunidad, y es más pobre, descendiente de linajes indios, mujer y adulta, está más "localizada" y su oferta de consumo cultural es muy reducida. Por el contrario, en el otro extremo, mientras uno está en Estados Unidos, y era varón, joven, con residencia y trabajo estable, más "globalizado" está, y sus ofertas para consumo cultural serán enormes. Sin embargo, aquí viene lo paradójico. Mientras la mujer "localizada" de San Antonio de Cocha querrá (sic) dejen de tener su suerte, empujándolos lo más pronto posible a la migración; el joven cochano en Estados Unidos construirá, ante el incremento de la oferta cultural y la discriminación ante una identidad que es crecientemente situacional y fronteriza, imágenes "esencialistas" en su memoria, recordando lo positivo de la comunidad y olvidando lo negativo de esta con el 
paso de los ańos. Lo cual lo llevará a incrementar el fenómeno urbano que hemos denominado como "localismo-regionalismo (168-169).

El estudio de Raúl Rosales (2008), desde la teoría de los estudios subalternos, estudia la etnohistoria urba$\mathrm{na}^{12}$ del "sector marginal-urbano" San Juan Masías, que sería, según el autor, "un pueblo ajeno", circunscrito dentro del bloque hegemónico de un distrito limeño: San Borja.

Para el autor, "la indiferencia social no solo se da en largas distancias geográficas, sino que se sustentan en "omisiones políticas y ciudadanas" que pueden darse en relación a cualquier lugar del país: tan cerca como tan lejos. Estas acciones y omisiones están registradas en la memoria individual y colectiva de los pobladores de San Juan Masías, mostrando una estrecha relación entre historia, cultura y poder" (Rosales 2008, 15).

Si la antropología en la ciudad precedente buscó migrantes del campo a la ciudad, el estudio de Rosales busca a los marginados de la "ciudad hegemónica". ¿Cuál es la diferencia? ¿Lo popular es también parte de los distritos considerados como modernos o de los que se tiene la imagen moderna? Para Rosales, San Borja representa uno de los distritos hegemónicos (San Isidro, Miraflores, Surco, La Molina, etc.) y asume que existe un "paradigma dual", por lo que su crítica deviene en que se ha estudiado solo la periferia, lo que en cierta medida es cierto, pero no se pregunta sobre la existencia de lo criollo ni dónde ni cómo están los criollos, asume que están en el centro de la ciudad. No estudia a los criollos ni a la clase media, menos a la clase hegemónica, busca a lo "marginados" que se encuentran en zonas "céntricas" o "sectores criollos". Considera de ese modo que hay un paso de la dualidad monolítica a la dualidad heterogénea, ya que espacios hegemónicos también comportan diferencias

12 "La etnohistoria urbana es un nuevo método de lectura de lectura del pasado para analizar los conflictos y tensiones del presente en la ciudad. Por este motivo, utilizo ésta metodología para analizar el caso del sector marginal-urbano San Juan Masías que es un pueblo ajeno dentro de San Borja. La construcción etnohistórica lo realicé inicialmente por medio del registro de testimonios en formato de historias de vida de los informantes. Estos relatos están enmarcados en la categoría de "interpretaciones de segundo orden", es decir, cuando el informante en el momento de contar o relatar una realidad social, política y cultural lo que está realizando, en el fondo es una interpretación del mundo que observa y experimenta. Esta es la propuesta de Clifford Geertz, quien considera que la verdad del relato se debe entender como ficciones: "en el sentido que son algo hecho, algo formado, compuesto --que es la significación de fictio- no necesariamente falsas o inefectivas meros experimentos mentales" (2000:28)”. (Rosales 2008, 16-17). y "marginados". No analiza los grandes cambios de configuración y uso espacial de su ámbito de estudio que hoy en día es otra realidad: una alta dinámica de intercambio económico, desarrollo comercial intenso y uso del espacio para un número considerable de instituciones estatales y privados.

Otras investigaciones más recientes comprenden los trabajos de Renato Merino Solari (2008) y del profesor Pedro Jacinto Pazos (2012). El estudio del profesor Pedro Jacinto fue publicado como libro el 2014 por el Fondo Editorial de la Universidad Nacional Mayor de San Marcos. No se conoce otro trabajo que haya tenido ese camino. Investiga la dinámica de los distintos actores sociales y sus nuevas formas de racionalizar los nuevos espacios que convierten en parte de la vida cotidiana de los pobladores. Estudia a los microempresarios y megamercados de Lima Norte y las transformaciones y configuraciones en términos de identidad, consumo y distinción. Su importancia radica en buscar comprender estas los sentidos de estas categorías más allá de los ámbitos "económicos-produccionistas", y considera la subjetividad e intersubjetividad en las percepciones de los microempresarios. Además, realiza un análisis comparativo con las dinámicas de los mercados de Europa, de manera especial Alemania.

Merino Solari, por su parte, realiza una "investigación de gabinete”, un análisis sobre discursos de los antropólogos acerca de los migrantes urbanos populares en Lima y la emergencia del sujeto democrático desde 1980 hasta 1992. Estudia la irrupción de un sujeto como agente principal de la praxis social y del devenir histórico, dando un giro de grupos marginales y potencialmente peligrosos ${ }^{13}$ para el orden existente a fundamentales para el desarrollo y progreso nacional; es decir, los migrantes se convirtieron en el agente principal del proceso democratizador y modernizador que experimentaba o debía experimentar el Perú. Se sitúa en el momento específico de su aparición, de su irrupción, de su transformación como idea hegemónica en las ciencias sociales. Sin embargo, el desarrollo posterior queda al margen. La investigación es realizada desde la perspectiva postestructural de Foucault, hace uso del método arqueológico de este último autor, de tal modo que su preocupación general está en analizar y reflexionar sobre los vínculos de las relaciones de poder y la producción teórica-práctica en las ciencias sociales. Y busca explicar los cambios socio-

13 Considera que este tipo de discursos y descripciones negativas de los migrantes de las barriadas fueron hechas sobre todo por parte de los economistas y sociólogos. 
culturales que propiciaron la emergencia y el desarrollo de esos discursos. Plantea que la emergencia y el desarrollo de los discursos académicos sobre los migrantes, constituyeron una construcción cultural realizada por los especialistas que permitió la instrumentalización y proyección de dicha realidad.

En sus términos, en relación esos cambios socioculturales y la configuración de zonas urbanas en el espacio principal de biopoder (principalmente Lima), dice lo siguiente:

El contexto sociocultural de este proceso fue los cambios que experimentó el Perú a partir de 1940: expansión del capitalismo (ampliación de las relaciones de importaciones, desarrollo de mercados internos, etc.), crisis de la agricultura andina (desequilibrio de la relación hombre-tierra), aumento demográfico, masivas migraciones del campo a la ciudad, urbanización del país, proyecto de modernización "desde arriba", que convirtieron a las zonas urbanas - especialmente a Lima- en el centro de las contradicciones sociopolíticas del país; es decir en el espacio decisivo de las luchas, por tanto, en el espacio principal del biopoder. De forma concomitante a estas dinámicas, la reflexión y producción académica se orientó hacia los migrantes andinos de la ciudad capital, convirtiéndolos en su referente empírico. En otros términos, la población urbana se convirtió en el principal objeto de exploración científica y de preocupación política”. (pp. 15-16).

Las modificaciones de las relaciones de poder globales y sus múltiples independencias con las relaciones de poder nacionales y locales permitieron la emergencia de los discursos académicos sobre el sujeto democratizador, es decir, fue una construcción teórica-práctica que al país le tocó experimentar debido a esos cambios múltiples Señalados. La conversión de los migrantes en sujetos políticos está ligada a un momento específico, comportando valores como modernos, democráticos y capitalistas. Según el autor, el migrante para los intelectuales se convirtió en el apéndice y posibilidad de una sociedad liberal incompleta, o sea, en agente de la democracia capitalista:

Los migrantes fueron convertidos en el elemento primordial que consagraba una historia iniciada con la fundación de la República liberal, que se mantenía incompleta, como una promesa y una posibilidad hasta el presente, por constituir una "nación inconclusa (p. 17).
De ese modo, Merino hace explícito que su interés central no es análisis de lo que decía el autor ni su "verdadero pensamiento sino el espacio en el cual decía. Tampoco fue parte de su interés la correspondencia con los hechos sino las relaciones que permitieron los enunciados y la validez de los discursos. "Por esta razón que no interpretamos un texto o un especialista de manera específica” (p. 20). La característica de la construcción del sujeto democrático es que fue un fenómeno interdisciplinario.

En síntesis, podemos decir que Merino es absorbido, modelado y prefigurado por las ideas y planteamientos de Michel Foucault, por lo que sus conclusiones se hacen incluso más previsibles a los planteados en las hipótesis. El estudio de representaciones y constructos teóricos sin considerar las condiciones materiales y sociales de los autores que produjeron esas representaciones resulta siendo incompleto para entender las transformaciones en las producciones antropológicas. Merino no se da cuenta de que la antropología incluso desde sus inicios tuvo una visión positiva de las barriadas y del sujeto migrante, a excepción de algunas ambigüedades de Matos Mar, quien sí tenía una visión positiva de las barriadas pero un sentido crítico de las migraciones (Sandoval 2001).

\section{A modo de conclusión: análisis general de las tesis y temas pendientes}

Indudablemente, los procesos de migración, adaptación del migrante y formación de las barriadas, han sido los temas más tratados en las investigaciones antropológicas de lo urbano en San Marcos. Se suman entre estas tendencias los análisis del rol que juegan las relaciones de parentesco y la red étnica en la adaptación y solución de las problemáticas económicas y sociales de los migrantes en la ciudad, así como la construcción de hábitat. Lo interesante en toda la producción es que la antropología de San Marcos empieza a estudiar las transformaciones culturales y sociales desde muy temprano en que se convierte en centro de formación institucionalizada, aunque imaginaron en principio que las estrategias de sus comunidades de origen se reflejaban en la ciudad. Sin embargo, analicemos las características de estas investigaciones así como la ausencia de temáticas.

La construcción del proceso de migración desde las comunidades de origen se encuentra ausente, todas las 
tesis constituyen una visión urbana de los Andes, es decir, la migración ha sido estudiada desde las ciudades. No existe ningún estudio que refiera a los no migrantes, tampoco ha existido una preocupación por plantearse ¿por qué migran unos y por qué no los demás?

La comprensión del dualismo campo/ciudad, de este modo, ha sido como una relación dicotómica y como un proceso de cambio de uno al otro, pero no ha sido estudiado el proceso de transición, la intermedia del cambio de uno al otro, lo que Víctor Turner denominó como la liminalidad, es decir, el estado transicional que puede determinar y comportar múltiples relaciones y comportamientos. Y dentro de este análisis de transformaciones y la relación entre urbe y sector rural, pues, existen pocas investigaciones, como de Tapia (1974), que analiza la transformación de Huancayo a partir de la lectura del concepto de proceso de urbanización dependiente de Manuel Castells, relacionando aspectos locales con condiciones nacionales e internacionales.

No hay ninguna referencia a los estudios de Henri Lefebvre (1974, 1978, 2013/1974) respecto a estas temáticas, a pesar de que por estas épocas — década de los setenta fundamentalmente- este autor había ya publicado trabajos importantes sobre la relación entre campo y ciudad, el derecho a la ciudad, una crítica a la vida cotidiana e incluso una teoría diferente sobre la forma de producir el espacio y las implicancias que produce este proceso. Esta ausencia tiene relación directa con el contexto social y el entorno académico que predominó en la Escuela de Antropología. La mayoría de los profesores de la escuela estaban relacionados más con literaturas norteamericanas, un poco de inglesas y poco menos con la literatura francesa, y de este reducido pues había marcadas tendencias de los docentes que excluían a autores ligados al marxismo o simplemente no se conocía la producción.

Por otro lado, a partir de la crítica a la antropología cultural e indigenista sobre la territorialización de las identidades, se ha descuidado estudiar la relación de los sujetos con el territorio y las múltiples implicancias de las luchas de los sujetos por el territorio. Se encuentra ausente el estudio de comportamientos y prácticas como el tráfico de tierras en zonas de expansión de las ciudades. Tampoco se ha estudiado el rol de las redes de vecindad y de parentesco en los espacios contiguos al tejido urbano conocidos como ampliaciones.

El centro de análisis ha sido Lima, lo que en el caso de migración queda implícita la idea de andinización de la ciudad limeña. El estudio de las ciudades de otras regiones ha quedado casi pendiente, son solo dos tesis estudian ciudades de otras regiones del país: el de Tapia (Huancayo, Junín) y el de Guillermo Gutiérrez (El Porvenir, Trujillo, La Libertad).

En cuanto a las influencias teóricas en las primeras décadas - cincuenta y sesenta - son producciones de influencia propiamente indigenista y culturalista. Y a diferencia de producciones posteriores, las tesis de la décadas de los setenta y ochenta, se caracterizan por una marcada tendencia teórica, incluso ideológica y política. Entre estas se encuentran tesis vinculadas a las propuestas marxista, histórico-funcionalista y análisis de redes sociales y étnicas. Desde la década del noventa, si bien algunas tesis mantienen una cierta tendencia teórica predominante, es notoria la presencia mayor del eclecticismo, una misma etnografía está marcada y construida a partir de lecturas a autores de diversas tendencias. ¿Cuál es adecuado: hacer una tesis desde una determinada perspectiva teórica o el eclecticismo? Es una pregunta que puede tener respuestas disímiles pero podemos formular la hipótesis que el eclecticismo tiene causas institucionales, es decir, parece ser producto de la ausencia de una comunidad académica consolidada, y su mayor reflejo se encuentra en que las tendencias teóricas que se pueden registrar en las diferentes producciones académicas, no han tenido continuidad e influencia en generaciones posteriores, por lo que los cortes de una etapa a otra, o incluso en la misma, han sido pronunciados.

Finalmente, el reto de la antropología está en estudiar los fenómenos que hoy constituyen parte de hábitos y estilos de vida y que al mismo tiempo estructura formas de relacionarse y vivir en la ciudad, ocupar el espacio, hacer política. En otros términos, investigar lo que hacen, piensan y dicen los que producen la ciudad.

\section{Referencias bibliográficas}

Adams Alegre, Norma Beatriz (1989). Organización campesina y migrantes en Lima: El caso de la comunidad de Huahuapuquio. Tesis para optar el título profesional de Licenciado en la especialidad de Antropología. Lima: UNMSM/Facultad de Ciencias Sociales.

Altamirano Rúa, Teófilo (1977). Identidades étnicas y formación de asociaciones regionales en Lima Metropolitana. Tesis para optar el Grado Académico de Doctor en 
Antropología. Lima: UNMSM/Programa Académico de Ciencia Social.

Arroyo Hurtado, Roberto (coord.) (2015). Lima Norte. Caracterización económica y proyecciones sociales. Lima: Fondo Editorial de la Universidad de Ciencias y Humanidades.

Arroyo Hurtado, Roberto (2014). Prólogo. En: Pedro Jacinto Pazos. Micronegocios vs. Megamercados. Otros sentidos de identidad, distinción y consumo en los microempresarios de Lima Norte. Lima: Fondo Editorial Universidad Nacional Mayor de San Marcos.

Ávila Molero, Javier (2001). Globalización y migración: El caso de los campesinos de la comunidad campesina de San Antonio de Cocha en Lima y los Estados Unidos. Tesis para optar el título de Licenciado en Antropología. Lima: UNMSM/Facultad d Ciencias Sociales.

BarReto Manunta, Giancarlo (2010). Descendientes italianos y ciudadanos italianos en la Lima de hoy. Un estudio sobre el incremento de ciudadanos italianos por derecho de jure sanguis en los años 2003-2007. Tesis para optar el grado de licenciado en Antropología. Lima: UNMSM/Facultad de Ciencias Sociales.

BeCERra Flores, Sonia (1977). Etnografia de un pueblo joven (Medalla Milagrosa). Tesis de Bachiller en Ciencias Sociales. Lima: UNMSM/Programa Académico de Ciencia Social.

Calderón, Julio (2006). Mercado de tierras urbanas, propiedad y pobreza. Lima: SINCO Editores.

Calderón, Julio (2005). La ciudad ilegal. Lima: UNMS.

Calderón, Julio (1998). "Mercado de tierras en áreas agrícolas periurbanas de Lima”. Debate Agrario, No 28, pp.122, Lima.

Calderón, Julio y Maquet, Claudio (1990). Las ideas urbanas en el Perú (1950-1989). Lima: Instituto de Desarrollo Urbano.

Chueca Goitia, Fernando (2016 [1968]). Historia del urbanismo. Madrid: Alianza Editorial.

Damonte (1994). Componentes de la cultura urbana en el Perú. En: Anthropológica, № 11. Lima: PUCP.

Degregori, Blondet y Lynch (1986). Conquistadores de un nuevo mundo. De invasores a ciudadanos. Lima: IEP.

Golte, Jürgen (1999). Campo y ciudad. Los intereses cambiantes de los antropólogos.

Golte, Jürgen y AdAms, Norma (1990 [1987]). Los caballos de Troya de los invasores. Estrategias campesinas en la conquista de la gran Lima. Los invasores. Lima: Instituto de Estudios Peruanos.

Gonzales Barbajal, Gabriela Leticia (1994). Estudio etnográfico sobre Lima prehispánica. Tesis para optar el tí- tulo profesional de Licenciado en Antropología. Lima: UNMSM/Facultad de Ciencias Sociales.

GoreliK, Adrián (2013). Miradas sobre Buenos Aires. Historia cultural y critica urbana. Buenos Aires: Siglo XXI.

Gutiérrez Chacón, Guillermo (1974). Análisis concreto de la situación concreta de la barriada de El Porvenir. Lima: UNMSM.

IzIGA NúŇEZ, Róger (1993). Sociología, movimientos sociales y espacio urbano. El caso de Lima Metropolitana. Lima: Universidad Nacional Mayor de San Marcos.

Jacinto Pazos, Pedro 2014 Micronegocios vs megamercados: Otros sentidos de identidad, distinción y consumo en los microempresarios de Lima Norte. Lima: Fondo Editorial UNMSM.

Jacinto Pazos, Pedro (2011). Pobreza extrema y exclusión socio-cultural en el Perú: Una mirada desde Chimbote. Lima: Fondo Editorial UNMSM.

Landa Vásquez, Ladislao (2004) Nos llaman y entramos. Los modos de participación en el espacio rural: Cusco y Apurimac. Lima: IEP.

Landa V., Ladislao (1992). Los caminos de la música. Los géneros populares andinos en un medio urbano. Tesis de Bachiller en Antropología. Lima: UNMSM/Facultad de Ciencias Sociales. 99 Pp.

Lefebvre, Henri (2013 [1974]). La producción del espacio. Madrid: Capitán Swing.

Lefebvre, Henri (1978). De lo rural a lo urbano. Barcelona: Ediciones Península.

Lefebvre, Henri (1974). La vida cotidiana en el mundo moderno. Madrid: Alianza Editorial.

Mathews Carmelino, Daniel (2016). La ciudad cantada (Lima, Santiago, Buenos Aires). Lima: CEDET.

Merino de Zela, E. Mildred (1958). El cerro San Cosme (formación de una barriada). Tesis para optar el grado de Bachiller. Lima: UNMSM/Instituto de Etnología y Arqueología.

Merino Solari, Renato Jorge (2008). Los discursos sobre los migrantes urbanos populares en Lima y la emergencia del sujeto democrático (1980-1992). Tesis para optar el Grado de Licenciado en Antropología. Lima: UNMSM/Facultad de Ciencias Sociales.

Pimentel Sánchez, Nekson (2014). El laberinto de la periurbanización y la cultura política en Carabayllo. En: Estructura Salvaje, Año 3, No 3. Lima: Lluvia Editores, pp. 55-72.

Quijano, Aníbal (2014). De la dependencia histórico-estructural a la colonialidadldescolonialidad del poder. Buenos Aires: CLACSO.

Quinteros Salazar, J. Walter (1965). Urbanización migra- 
cional y aculturación (Estudio de la población obrera de la Molina). Tesis de Bachiller. Lima: UNMSM/Departamento de Etnología y Arqueología.

Quispe Pastrana, Edgar y Santiago Tácunan Bonifacio (2011). Carabayllo: Génesis de Lima Norte. Lima: Municipalidad de Carabayllo.

Riofrío, Gustavo (1991). Producir la ciudad (popular). Lima: Desco.

Ríos Espinoza, Gloria (2000). Cuidados de la salud del niño en un área urbano marginal. Una visión desde la madre migrante. Tesis para optar el título de Licenciada en Antropología. Lima: UNMSM/ Facultad de Ciencias Sociales.

Rosales León, Raúl Fernando (2008). Para ver más allá de lo evidente. Etnohistoria urbana, cultura y poder en el sector marginal-urbano San Juan Masias, San Borja. Lima: 1972-2006. Tesis para optar el título de Licenciado en Antropología. Lima: UNMSM/Facultad de Ciencias Sociales. 365 pp.

Salomón Zugbi, Alejandro Faruk (1972). "La invasión de las pampas de Collique y la formación del Pueblo Joven "Año Nuevo" después de la Ley 13517". Tesis para optar
Grado de Bachiller en Antropología. Lima: UNMSM/ Programa Académico de Ciencia Social.

Sánchez León, Abelardo (1990). Problemas y estudios urbanos en el Perú. En: Fernando Carrión (ed.). La investigación urbana en América Latina. Caminos recorridos y por recorrer. Estudios nacionales. Quito: Ciudad.

Tanaka, Martín (coord.) (2016). Antología del pensamiento crítico peruano contemporáneo. Buenos Aires: CLACSO.

Tanaka, Martín (1999). Los partidos políticos en el Perú. 1992.1999: Estabilidad, sobrevivencia y política mediática. Lima: Instituto de Estudios Peruanos. Documento de trabajo $\mathrm{n}^{\circ} 108$

TAPIA TAKeY, María Ysabel (1976). El proceso de urbanización de Huancayo entre los años 1900-1975. Tesis para optar el Grado Académico de Bachiller en Ciencias Sociales. Lima: UNMSM/ Dirección de Programas Académicos de Ciencia Social, Antropología. 107 pp.

Vallejos Burgos (1996). Adaptación del migrante rural a la urbe metropolitana. Tesis doctoral. Lima: UNMSM/Facultad de Ciencias Sociales. 